\title{
LARGE-SCALE ASYMMETRIES IN THE TRANSITIONAL DISKS OF SAO 206462 AND SR 21
}

\author{
Laura M. Pérez ${ }^{1,3}$, Andrea Isella ${ }^{2}$, John M. Carpenter ${ }^{2}$, and Claire J. Chandler ${ }^{1}$ \\ ${ }^{1}$ National Radio Astronomy Observatory, P.O. Box O, Socorro, NM 87801, USA \\ ${ }^{2}$ California Institute of Technology, 1200 East California Boulevard, Pasadena, CA 91125, USA \\ Received 2013 December 10; accepted 2014 February 3; published 2014 February 14
}

\begin{abstract}
We present Atacama Large Millimeter/submillimeter Array (ALMA) observations in the dust continuum (690 GHz, $0.45 \mathrm{~mm}$ ) and ${ }^{12} \mathrm{CO} J=6-5$ spectral line emission of the transitional disks surrounding the stars SAO 206462 and SR 21. These ALMA observations resolve the dust-depleted disk cavities and extended gaseous disks, revealing large-scale asymmetries in the dust emission of both disks. We modeled these disk structures with a ring and an azimuthal Gaussian, where the azimuthal Gaussian is motivated by the steady-state vortex solution from Lyra \& Lin. Compared to recent observations of HD 142527, Oph IRS 48, and LkH $\alpha$ 330, these are low-contrast ( 2 ) asymmetries. Nevertheless, a ring alone is not a good fit, and the addition of a vortex prescription describes these data much better. The asymmetric component encompasses $15 \%$ and $28 \%$ of the total disk emission in SAO 206462 and SR 21, respectively, which corresponds to a lower limit of $2 M_{\text {Jup }}$ of material within the asymmetry for both disks. Although the contrast in the dust asymmetry is low, we find that the turbulent velocity inside it must be large ( $\sim 20 \%$ of the sound speed) in order to drive these azimuthally wide and radially narrow vortex-like structures. We obtain residuals from the ring and vortex fitting that are still significant, tracing non-axisymmetric emission in both disks. We compared these submillimeter observations with recently published $H$-band scattered light observations. For SR 21 the scattered light emission is distributed quite differently from the submillimeter continuum emission, while for SAO 206462 the submillimeter residuals are suggestive of spiral-like structure similar to the near-IR emission.
\end{abstract}

Key word: protoplanetary disks

Online-only material: color figures

\section{INTRODUCTION}

The structure of a circumstellar disk is expected to dramatically change during the planet formation process. In a few million years an optically thick massive protoplanetary disk will transform into an optically thin debris disk with little material (e.g., Hernández et al. 2007). Processes like viscous accretion, photo evaporation, grain growth, and planet formation shape the structure of circumstellar disks (Clarke et al. 2001; Dullemond \& Dominik 2005; Ireland \& Kraus 2008), and in particular, the process of planet formation creates gaps, cavities, and asymmetries that can be directly observed (Wolf \& D'Angelo 2005). Circumstellar disks with gaps and/or cavities lack warm dust close to the star while still possessing a significant reservoir of cold dust in the outer disk. Such disks are classified as transitional disks, and much is known about their inner disk gaps/cavities (e.g., Dutrey et al. 2008; Salyk et al. 2009; Espaillat et al. 2010; Isella et al. 2012). However, it is only recently that observations have reached the sensitivity and angular resolution required to study asymmetrical features in the outer disk structure. Particularly, the only known examples of high-contrast asymmetries in transitional disks at submillimeter wavelengths are HD 142527 (Casassus et al. 2013; Fukagawa et al. 2013), Oph IRS 48 (van der Marel et al. 2013), and $\mathrm{LkH} \alpha 330$ (Isella et al. 2013). To understand the process of planet formation, long-wavelength observations are critical, since they are less affected by optical depth, and hence trace the bulk of the mass surface density.

In this Letter, we present Atacama Large Millimeter/ submillimeter Array (ALMA) observations at $0.45 \mathrm{~mm}$ of two disks surrounding the stars SAO 206462 and SR 21, which exhibit large-scale asymmetries in their dust continuum

\footnotetext{
3 Jansky Fellow.
}

emission that were not known before with high significance. SAO $206462^{4}$ is an isolated Herbig Ae/Be star (F4Ve, $\left.1.7 M_{\odot}\right)$, at a distance of $142 \mathrm{pc}$ in the Sco-OB2 association (Müller et al. 2011). The disk surrounding SAO 206462 is classified as a transitional disk, with a large dust-depleted cavity $\left(R_{\text {cav }}=46 \mathrm{AU}\right)$ and a massive outer disk (Andrews et al. 2011; Brown et al. 2009). Recently, scattered light observations of the polarized intensity at near-IR wavelengths revealed spiral-like structure in the outer disk of SAO 206462 (Muto et al. 2012; Garufi et al. 2013). SR 21 is a young G3 star $\left(2.5 M_{\odot}\right)$ in the $\rho$-Ophiuchus star forming region at a distance of 119 pc (Lombardi et al. $2008)$. The dust-depleted cavity ( $R_{\mathrm{cav}}=36 \mathrm{AU}$, Andrews et al. 2011) in this transition disk (Brown et al. 2007, 2009) is not completely devoid of material, as rovibrational $\mathrm{CO}$ line observations reveal the presence of inner disk gas (Pontoppidan et al. 2008; Brown et al. 2013). Furthermore, recent polarized intensity $H$-band observations of SR 21 by Follette et al. (2013) display emission inside the cavity radius, and unlike SAO 206462, the radial profile of emission is quite smooth.

\section{OBSERVATIONS AND CALIBRATION}

Observations at $0.45 \mathrm{~mm}(690 \mathrm{GHz})$ of SAO 206462 and SR 21 were obtained during ALMA Cycle 0 between 2012 June-July, with baselines ranging from 50 to $500 \mathrm{~m}$. Four spectral windows were configured to provide $1.875 \mathrm{GHz}$ of bandwidth per polarization, 3840 channels of $0.488 \mathrm{MHz}$ width $\left(\sim 0.2 \mathrm{~km} \mathrm{~s}^{-1}\right)$, for a total bandwidth of $7.5 \mathrm{GHz}$ in dual polarization. The bandpass response was calibrated with 3C 279 observations, the absolute flux scale was measured using Titan observations, and the phase and amplitude gains were tracked by periodically observing J1427-4206 and J1625-2527 for

\footnotetext{
4 Also HD 135344B.
} 


\section{SAO 206462}
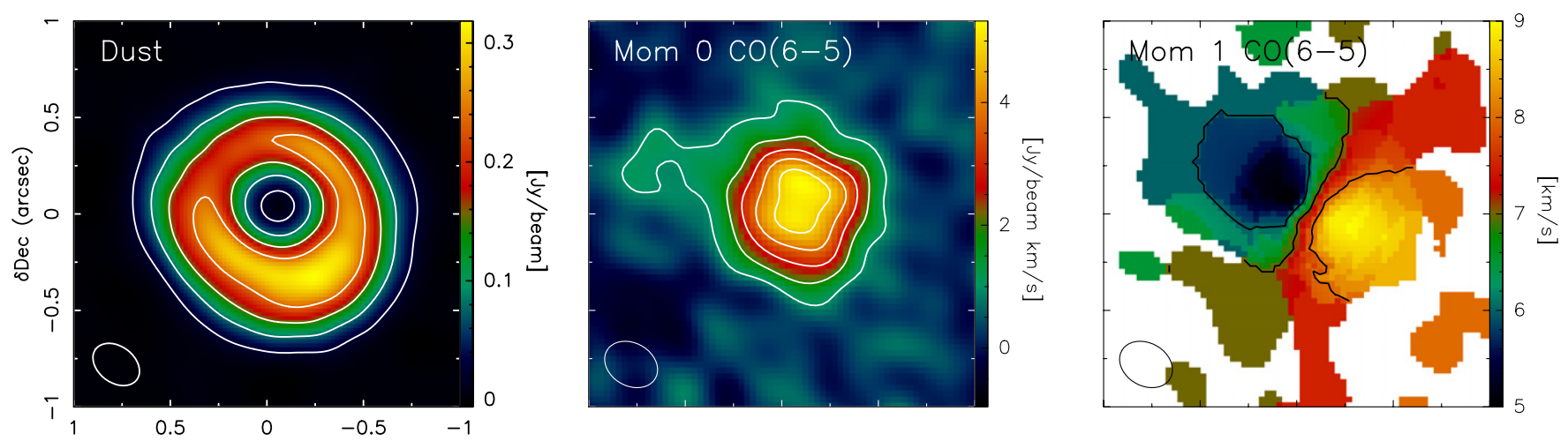

\section{SR 21}
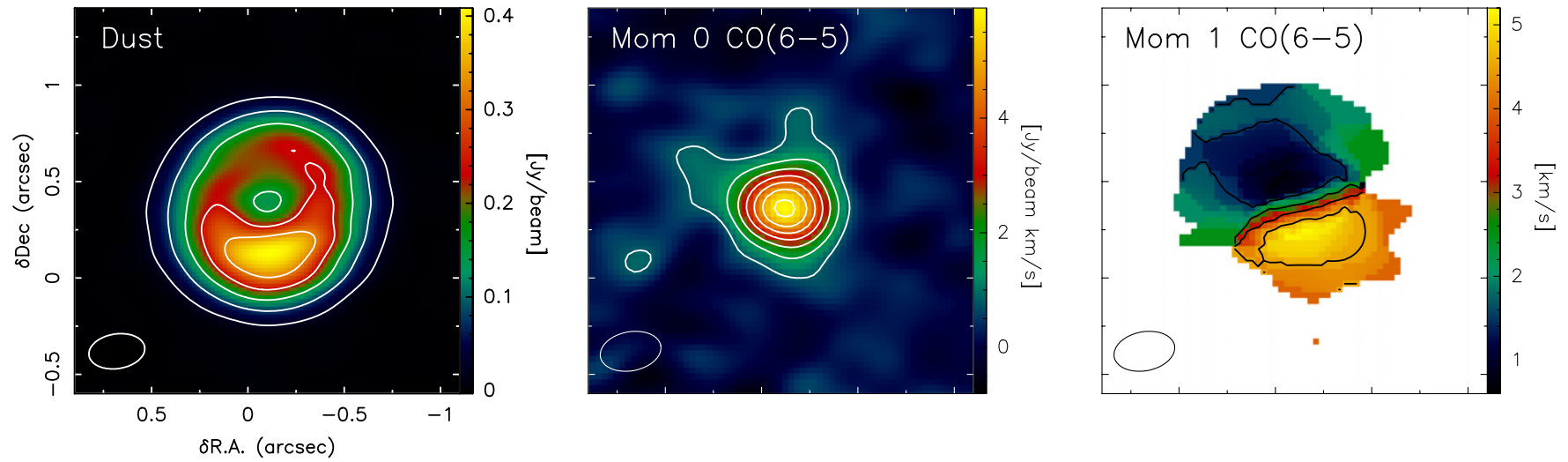

Figure 1. ALMA observations of dust and gas emission for the disks surrounding SAO 206462 (top row) and SR 21 (bottom row). Ellipses indicate beam sizes listed in Table 1. Left panels: dust continuum emission (color scale) and dust brightness temperature (contours starting at $10 \mathrm{~K}$, spaced by $5 \mathrm{~K}$ ). Middle panels: ${ }^{12} \mathrm{CO} J=6-5$ moment 0 map, contours start and are spaced by $3 \sigma$, where $\sigma$ is the rms noise level in the map. Right panels: ${ }^{12} \mathrm{CO} J=6-5$ moment 1 map, contours are spaced by $1 \mathrm{~km} \mathrm{~s}^{-1}$.

(A color version of this figure is available in the online journal.)

Table 1

Image Properties for Dust Continuum and Spectral Line Observations

\begin{tabular}{|c|c|c|c|c|c|c|c|c|c|}
\hline \multirow[t]{2}{*}{ Target } & \multirow{2}{*}{$\begin{array}{l}\text { R.A. }^{a} \\
(\mathrm{~J} 2000)\end{array}$} & \multirow{2}{*}{$\begin{array}{c}\mathrm{Dec}^{\mathrm{a}} \\
(\mathrm{J} 2000)\end{array}$} & \multicolumn{4}{|c|}{ Continuum } & \multicolumn{3}{|c|}{ Spectral Line } \\
\hline & & & $\begin{array}{l}S_{v}{ }^{\mathrm{b}} \\
(\mathrm{Jy})\end{array}$ & $\begin{array}{c}\mathrm{rms} \\
\left(\mathrm{Jy} \mathrm{beam}^{-1}\right)\end{array}$ & $\begin{array}{c}\text { Beam } \\
\text { Size }\end{array}$ & $\begin{array}{l}\text { Beam } \\
\text { P.A. }\end{array}$ & $\begin{array}{c}\mathrm{RMS}^{\mathrm{c}} \\
\left(\mathrm{Jy} \mathrm{beam}^{-1}\right)\end{array}$ & $\begin{array}{l}\text { Beam } \\
\text { Size }\end{array}$ & $\begin{array}{l}\text { Beam } \\
\text { P.A. }\end{array}$ \\
\hline SR 21 & $16: 27: 10.281$ & $-24: 19: 12.88$ & 3.3 & 0.0011 & $0 ! 29 \times 00^{\prime \prime} 18$ & $-81^{\circ}$ & 0.07 & $0^{\prime \prime} 32 \times 00^{\prime \prime} 20$ & $-79^{\circ}$ \\
\hline
\end{tabular}

Notes.

${ }^{\text {a }}$ Phase center from the Roeser et al. (2010) catalog, including proper motion (epoch J2000).

${ }^{\mathrm{b}}$ Flux density integrated inside aperture where emission is above $3 \times \mathrm{rms}$ noise level.

${ }^{\mathrm{c}} \mathrm{rms}$ noise level in a $0.5 \mathrm{~km} \mathrm{~s}^{-1}$ channel.

SAO 206462 and SR 21, respectively. A total of 25 minutes on-source time was obtained for both targets. Visibilities were calibrated and imaged in CASA. Given the high signal-to-noise ratio of these observations amplitude and phase self-calibration was performed after standard phase referencing. Table 1 lists observational properties of the continuum and spectral line maps, obtained using Briggs weighting with a robust of 0.5 .

\section{OBSERVATIONAL RESULTS}

We present maps of the dust and gas emission $\left({ }^{12} \mathrm{CO} \mathrm{J}=\right.$ 6-5) in Figure 1. These observations resolve the structure of each transitional disk, revealing for the first time the striking morphology in the dust continuum emission for SAO 206462 and SR 21: a non-uniform ring with a bright asymmetry located in the southwest for SAO 206462 and in the south for SR 21. The integrated intensity map (moment 0 ) and the intensity-weighted mean velocity map (moment 1 ) of ${ }^{12} \mathrm{CO} J=6-5$ are presented in the middle and right panels of Figure 1. In contrast with the dust emission, the gaseous component from the ${ }^{12} \mathrm{CO}$ moment 0 map appears to be quite compact and mostly symmetric. However, significant emission $(>3 \sigma)$ can be found at large radii in the ${ }^{12} \mathrm{CO}$ moment 1 map, just as with the dust emission. Since emission from ${ }^{12} \mathrm{CO}$ is most likely optically thick, it is not a good tracer of depletion inside the cavity and other tracers must be used (Bruderer 2013). However, a large depletion of the inner disk ( $>10^{6}$; Bruderer 2013) can give rise to the double-peaked structure observed in the SAO 206462 moment 0 map. Further 
Table 2

Best-fit Results

\begin{tabular}{|c|c|c|c|c|c|c|}
\hline & & & \multicolumn{2}{|c|}{ SAO 206462} & \multicolumn{2}{|c|}{ SR 21} \\
\hline & & & $\begin{array}{c}\text { Ring } \\
\chi_{\text {red }}^{2}=1.18\end{array}$ & $\begin{array}{c}\text { Ring }+ \text { Vortex } \\
\chi_{\text {red }}^{2}=1.05\end{array}$ & $\begin{array}{c}\text { Ring } \\
\chi_{\text {red }}^{2}=1.39\end{array}$ & $\begin{array}{c}\text { Ring }+ \text { Vortex } \\
\chi_{\text {red }}^{2}=1.06\end{array}$ \\
\hline Ring & $F_{R}$ & $(\mu \mathrm{Jy})$ & $5.72 \pm 0.01$ & $5.24_{-0.01}^{+0.03}$ & $5.91 \pm 0.01$ & $4.72 \pm 0.02$ \\
\hline \multirow[t]{2}{*}{ Parameters } & $r_{R}$ & $(\mathrm{AU})$ & $59.9 \pm 0.1$ & $65.3 \pm 0.1$ & $36.4 \pm 0.1$ & $35.0 \pm 0.1$ \\
\hline & $\sigma_{R}$ & $(\mathrm{AU})$ & $18.0 \pm 0.1$ & $15.5 \pm 0.1$ & $14.90 \pm 0.04$ & $13.9 \pm 0.1$ \\
\hline Vortex & $F_{V}$ & $(\mu \mathrm{Jy})$ & $\ldots$ & $7.3_{-0.3}^{+0.1}$ & $\ldots$ & $3.91_{-0.03}^{+0.02}$ \\
\hline \multirow[t]{4}{*}{ Parameters } & $r_{V}$ & $(\mathrm{AU})$ & $\cdots$ & $41.5_{-0.1}^{+0.2}$ & $\cdots$ & $46.0_{-0.1}^{+0.2}$ \\
\hline & $\theta_{V}$ & $\left({ }^{\circ}\right)$ & $\cdots$ & $193.9_{-0.6}^{+0.4}$ & $\cdots$ & $177.7_{-0.3}^{+0.5}$ \\
\hline & $\sigma_{r, V}$ & $(\mathrm{AU})$ & $\cdots$ & $6.6_{-0.1}^{+0.3}$ & $\cdots$ & $14.4_{-0.1}^{+0.2}$ \\
\hline & $\sigma_{\theta, V}$ & $(\mathrm{AU})$ & $\cdots$ & $46.7_{-0.4}^{+0.7}$ & $\cdots$ & $40.4 \pm 0.4$ \\
\hline \multirow[t]{3}{*}{ Disk geometry } & $i$ & $\left({ }^{\circ}\right)$ & $22.9_{-0.2}^{+0.3}$ & $22.8_{-0.3}^{+0.2}$ & $15.0_{-0.4}^{+0.3}$ & 15 (fixed) \\
\hline & $x_{0}$ & (mas) & $-44.1 \pm 0.4$ & $-50.0_{-0.4}^{+0.6}$ & $-12.5 \pm 0.4$ & $-17.6_{-0.6}^{+0.4}$ \\
\hline & $y_{0}$ & (mas) & $-51.6 \pm 0.5$ & $-75.0_{-0.2}^{+0.8}$ & $-40.2 \pm 0.3$ & $2.7_{-0.7}^{+1.1}$ \\
\hline
\end{tabular}

analysis of these $\mathrm{CO}$ observations will be deferred to a future publication. We measured the disk position angle (P.A.) from the rotation of the disk in the ${ }^{12} \mathrm{CO}$ moment 1 map; the measured P.A. east of north is $\sim 62^{\circ}$ and $\sim 16^{\circ}$ for SAO 206462 and SR 21 , respectively. Our derived P.A. is consistent with measurements found in the literature. For SAO 206462 measurements from molecular gas (inside and outside the cavity) and polarization give a P.A. between $55^{\circ}-64^{\circ}$ (Pontoppidan et al. 2008; Lyo et al. 2011; Muto et al. 2012; Andrews et al. 2011). For SR 21, Pontoppidan et al. (2008) constrained its P.A. to $16^{\circ} \pm 3^{\circ}$ from rovibrational $\mathrm{CO}$ band observations of molecular gas inside the cavity, while Brown et al. (2009) inferred a P.A. of $\sim 15^{\circ}$ from dust continuum observations of the outer disk.

The contrast between the peak of emission and the opposite side of the disk is $1.5 \pm 0.02$ in SAO 206462 and $1.9 \pm 0.01$ in SR 21. These asymmetries have lower contrast values than those found in $\mathrm{LkH} \alpha 330$ ( $\times 3$; Isella et al. 2013), HD 142527 ( $\times 30$; Casassus et al. 2013; Fukagawa et al. 2013), and Oph IRS 48 ( $\times 130$; van der Marel et al. 2013); nevertheless, they are significant enough to warrant further analysis.

The $850 \mu \mathrm{m}$ observations from Andrews et al. (2011) constrained the surface density at the edge of the cavity to be $\Sigma_{\text {dust }} \sim 0.1 \mathrm{gm} \mathrm{cm}^{-2}$ for both SAO 206462 and SR 21. Assuming a dust opacity of $\kappa_{v}=10 \mathrm{~cm}^{2} \mathrm{~g}^{-1}$ at $450 \mu \mathrm{m}$ (Beckwith et al. 1990), we find that the $0.45 \mathrm{~mm}$ optical depth of dust is $\tau \sim 1$ at the peak of the emission from the ring, and it is expected that at larger radii from the disk $\tau$ should be lower. We also computed the brightness temperature $\left(T_{b}\right)$ of the emission without the Raleigh-Jeans approximation. The left panels of Figure 1 show contours of $T_{b}$, starting at $10 \mathrm{~K}$ and spaced every $5 \mathrm{~K}$. At the peak of emission, $T_{b} \sim 30 \mathrm{~K}$ for SAO 206462, and $T_{b} \sim 35 \mathrm{~K}$ for SR 21 . At the distance where the asymmetry is located ( 50 AU for SAO 206462 and $\sim 40$ AU for SR 21), a temperature of $30-35 \mathrm{~K}$ is a reasonable value for the dust temperature in a flared disk, consistent with $\tau \approx 1$ derived above. We expect then that these ALMA observations trace some of the mass surface density, since the emission is borderline between optically thin and optically thick emission.

\section{ANALYSIS}

To constrain the structure of these large-scale asymmetries, we compare the dust continuum observations of SAO 206462 and SR 21 with simple morphological models that describe the emission from a ring and a vortex. These morphological models were chosen since these are known transitional disks that can be well-described by a ring-like structure (Andrews et al. 2011; Brown et al. 2009), and theoretical studies suggest that asymmetries in disk emission may arise for vortices (e.g., Regály et al. 2012). For the ring morphology we use a Gaussian prescription in the radial direction: in polar coordinates ${ }^{5}$ the ring emission corresponds to $F(r, \theta)=F_{R}(\theta) e^{-\left(r-r_{R}\right)^{2} / 2 \sigma_{R}^{2}}$, where $r_{R}$ is the radius where the ring peaks, $F_{R}$ is the flux density at $r_{R}$, and $\sigma_{R}$ is the ring width. In our modeling the ring emission is assumed to be axisymmetric. For the vortex morphology, we employ the prescription presented in Lyra \& Lin (2013), where the mass surface density of a steady-state vortex can be described as a Gaussian in the radial and azimuthal directions: $F(r, \theta)=F_{V} e^{-\left(r-r_{V}\right)^{2} / 2 \sigma_{r, V}^{2}} e^{-\left(\theta-\theta_{V}\right)^{2} / 2 \sigma_{\theta, V}^{2}}$, where $r_{V}$ and $\theta_{V}$ are the radius and P.A. of the vortex's peak, $F_{V}$ is the flux density at $r_{V}, \theta_{V}$, and $\sigma_{\theta, V}, \sigma_{r, V}$ are the radial and azimuthal width of the vortex. This way, the structure of a disk can be described by a ring of emission (three parameters), a vortex (five parameters), or the combination of both (eight parameters).

We fix the disk P.A. to the value measured in Section 3, and setup models of a ring and a ring with a vortex, leaving the disk center $\left(x_{0}, y_{0}\right)$ and inclination $(i)$ as free parameters. Each model realization is Fourier transformed and sampled at the same $u v$-positions as the observation. The best-fit model was found by minimizing the $\chi^{2}$ statistic using the latest implementation of the affine-invariant MCMC emcee software (Foreman-Mackey et al. 2013).

Table 2 presents the best-fit parameters found for a ring model and a ring+vortex model. As expected, the $\chi^{2}$ analysis favors a ring with a vortex model, since a ring alone cannot reproduce the large asymmetrical structures of these ALMA observations. We note that for SR 21, our preliminary ring+vortex model fitting favored a face-on geometry $\left(i \approx 0^{\circ}\right)$. Nevertheless, gas tracers show disk rotation making such geometry unlikely. Thus, we fixed the inclination to $i=15^{\circ}$ for SR 21 ring+vortex modeling, consistent with the ${ }^{12} \mathrm{CO} J=6-5$ line shape and motivated by the result of our ring-only model.

The best-fit model and residual maps for the two morphologies explored are presented in Figures 2 and 3 for SAO 206462

5 Where $r=\sqrt{x^{2}+y^{2}}, \theta=\tan (x / y)$. 

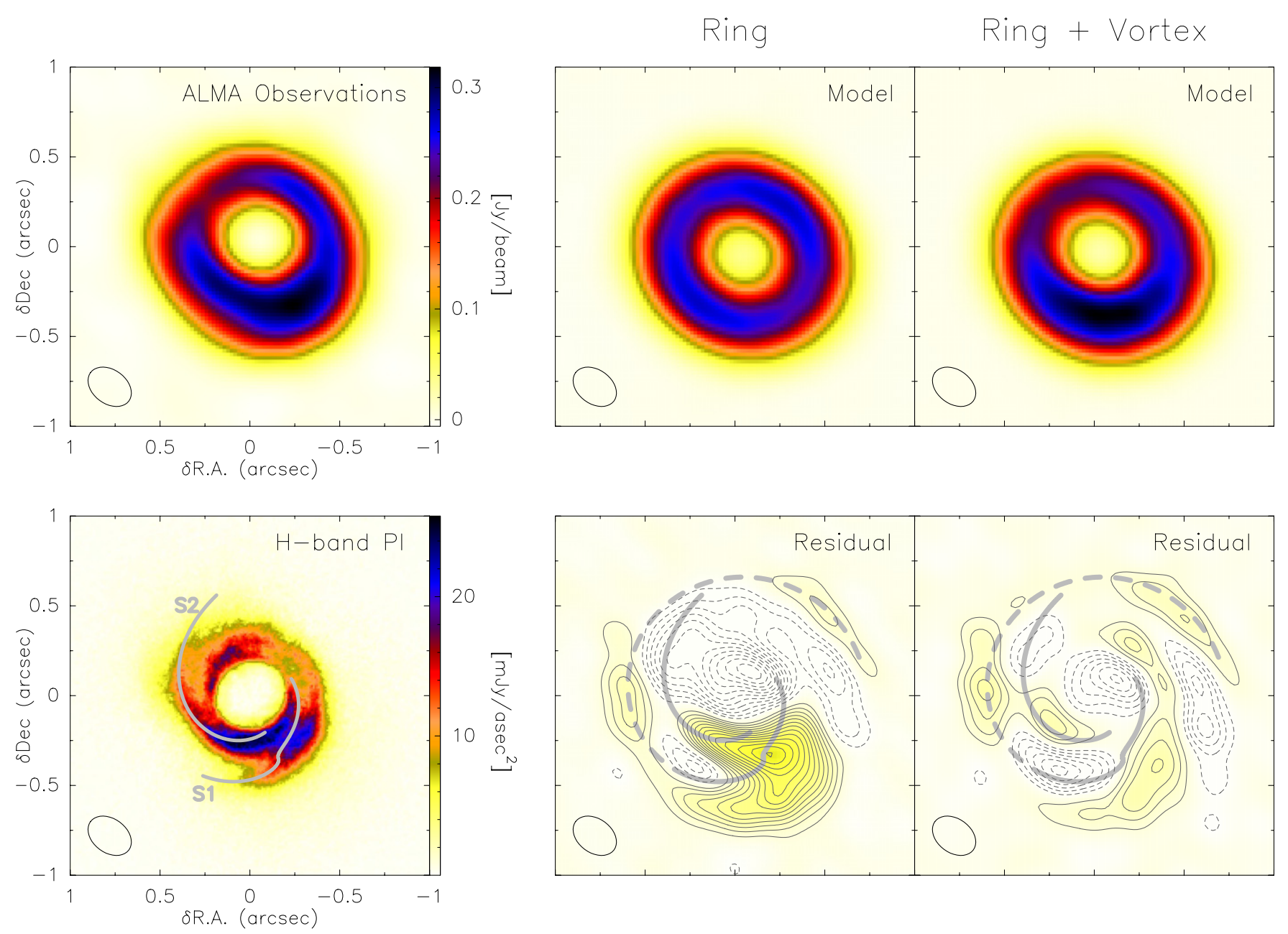

Figure 2. SAO 206462 ALMA observations (top left panel) compared with best-fit models for a ring (middle panels) and a ring with a vortex prescription (right panels). Residual maps were produced by subtracting the best-fit model from the SAO 206462 ALMA observations. Contours start at $\pm 3 \sigma$, successively spaced by $\pm 3 \sigma$. Ellipses indicate beam size and colorbar defines scale used. Bottom row, left panel: $H$-band polarized intensity image and the best-fit spiral arms from Muto et al. (2012). Spirals S1 and S2 also shown with grey lines in residual maps.

(A color version of this figure is available in the online journal.)

and SR 21, respectively. These figures compare ALMA observations (top row, leftmost panel) with the best-fit model and residual maps (middle and right panels), where the residual emission is found by subtracting the model from the observations. When considering only a ring, the best-fit model corresponds to the average flux of the disk over all azimuthal angles. Hence large regions of negative emission are expected since the azimuthally averaged flux also encompasses flux from the large-scale asymmetry. The addition of a vortex prescription in the fitting improves the resulting residual map and $\chi^{2}$ of the fit, although significant emission $(\gtrsim 12 \sigma)$ remains.

\section{DISCUSSION}

The cavity radius $\left(R_{\text {cav }}\right)$ found in the literature for these disks has some scatter, on average $R_{\text {cav }} \sim 45 \mathrm{AU}$ for SAO 206462 and $R_{\text {cav }} \sim 35 \mathrm{AU}$ for SR 21 (Brown et al. 2009; Andrews et al. 2009, 2011; Lyo et al. 2011). We find that $R_{\text {cav }}$ from these studies is consistent with our constraints within $r_{R} \pm \sigma_{R}$ for all but the Brown et al. (2009) observations of SAO 206462, which have lower angular resolution than these ALMA observations.

The analysis presented above demonstrates that a ring of emission does not describe the disk structure of SAO 206462 and SR 21; the addition of a vortex prescription describes the large-scale asymmetries better. However, the observed asymmetries might not directly trace the mass surface density, since short-wavelength observations may not be completely optically thin. Future long-wavelength observations will confirm that these asymmetries correspond to mass rather than temperature variations.

The vortex encompasses $15 \%$ of the total flux density for SAO 206462 and $28 \%$ for SR 21 . These are quite similar to the recently studied disk around $\mathrm{LkH} \alpha 330(30 \%)$ but much less dramatic than the Oph IRS 48 and HD 142527 disks, where a horseshoe shape, rather than a ring, is observed at millimeter wavelengths. The flux density in the asymmetry corresponds to a mass (dust+gas) of $\sim 2 M_{\text {Jup }}$ for both SAO 206462 and SR 21, assuming a temperature of $T=35 \mathrm{~K}$, a gas-to-dust ratio of 100 , and a dust opacity of $\kappa_{v}=10 \mathrm{~cm}^{2} \mathrm{~g}^{-1}$ at $450 \mu \mathrm{m}$ (Beckwith et al. 1990). These are lower limits on the vortex mass, given the uncertainty in optical depth at this wavelength. Compared to recent examples of large-scale asymmetries observed in $\mathrm{LkH} \alpha$ 330 and Oph IRS 48, the radial extent of the vortices in SAO 206462 and SR 21 is also quite narrow, with a FWHM of few tens of AU (see Table 2). Azimuthally, the vortices are well resolved, and while the SR 21 asymmetry has a FWHM $\sim 120^{\circ}$, similar to $\mathrm{LkH} \alpha 330$ and Oph IRS 48, the azimuthal extent in SAO 206462 is $30 \%$ larger, covering almost half of the disk. 

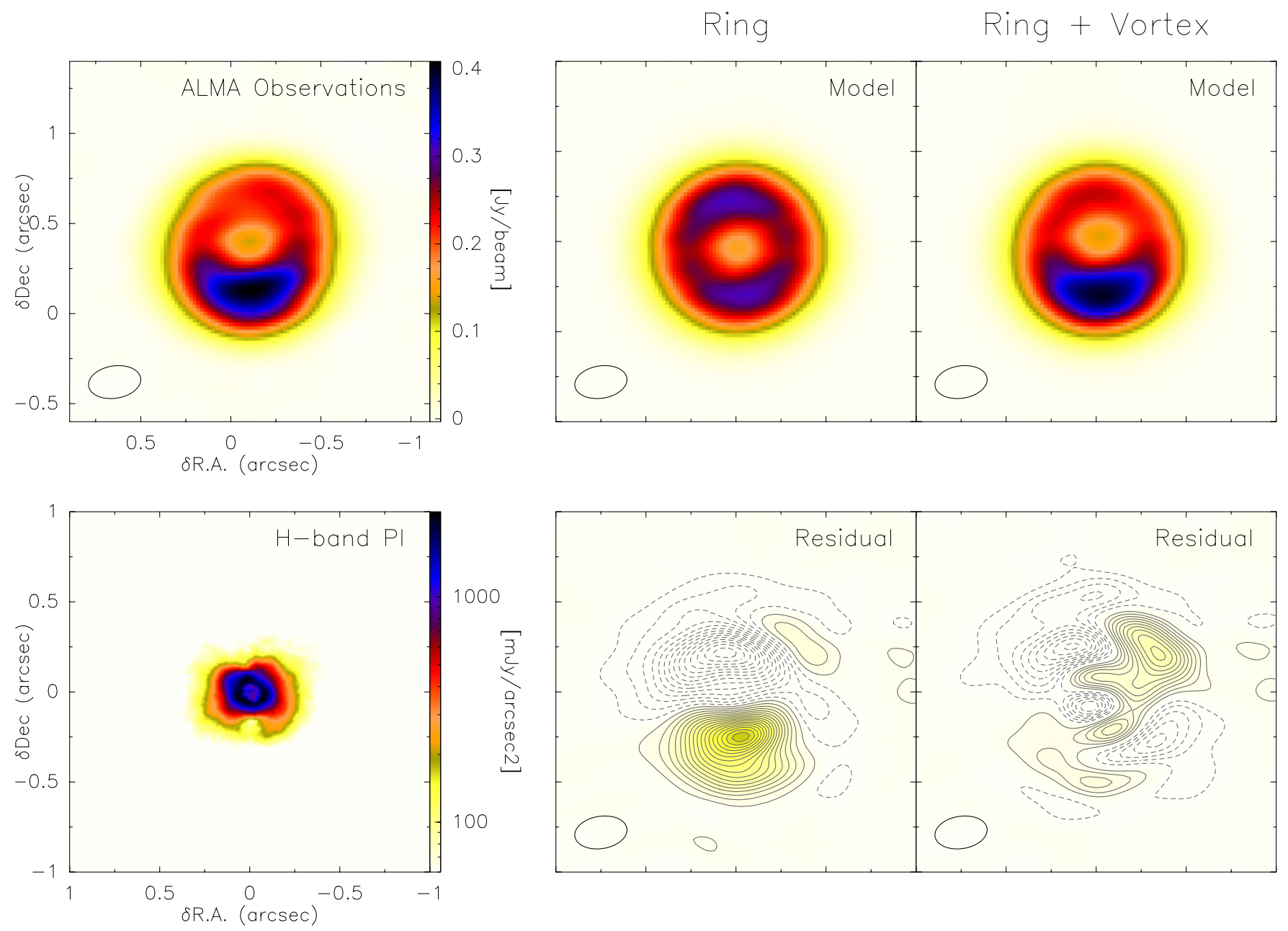

Figure 3. SR 21 ALMA observations (top left panel) compared with best-fit models for a ring (middle panels) and a ring with a vortex prescription (right panels). Residual maps were produced by subtracting the best-fit model from the SR 21 ALMA observations. Contours start at $\pm 3 \sigma$, successively spaced by $\pm 3 \sigma$ ( $\pm 6 \sigma$ for ring residual). Ellipses indicate beam size and colorbar defines scale used. Bottom row, left panel: $H$-band polarized intensity image from Follette et al. (2013).

(A color version of this figure is available in the online journal.)

From our modeling we find that the vortex aspect ratio is $\sigma_{\phi, V} / \sigma_{r, V}=7.1$ for SAO 206462 and $\sigma_{\phi, V} / \sigma_{r, V}=2.8$ for SR 21. Following the solution presented by Lyra \& Lin (2013), a steady-state vortex tends to concentrate large grains toward its center, in an amount that depends on the ratio between the dust grain Stokes number $(S t)$ and the gas turbulence velocity (see also Birnstiel et al. 2013). Our measurements of the vortex structure and knowledge of the Stokes number allow us to constrain the gas turbulence. Dust grains with size $a=1 \mathrm{~mm}$ and internal density $\rho_{s}=1 \mathrm{~g} \mathrm{~cm}^{-3}$, embedded in a disk with a surface density of $\Sigma=10 \mathrm{~g} \mathrm{~cm}^{-2}$ have a Stokes number $S_{t} \sim \rho_{s} a / \Sigma \sim 10^{-2}$. The vortex aspect ratio derived for SAO 206462 and SR 21 would then require turbulent velocities of about $90 \mathrm{~m} \mathrm{~s}^{-1}$ and $68 \mathrm{~m} \mathrm{~s}^{-1}$ respectively, corresponding to about $22 \%$ and $16 \%$ of the local isothermal sound speed for a gas temperature of $50 \mathrm{~K}$. Such values are about five times larger that those derived for Oph IRS 48, and consistent with an active disk layer due to the magnetorotational instability (Simon et al. 2011).

When including the vortex prescription in the fit, there are still significant residual emission in both SAO 206462 and SR 21. These residuals are not symmetric, and their morphology is not well described by an additional vortex since they extends outwards with increasing radius. A spiral-like structure may be a good approximation for the residual emission seen in both disks, but the number of parameters of such a prescription is quite large (at least five more parameters per spiral arm). Given the low signal of these features and degeneracies between such large number of parameters, we decided not to proceed with such a fit. Observations at higher angular resolution are required to study in greater detail the morphology of these structures.

On the bottom left panel of Figures 2 and 3, we present polarized intensity maps at $H$-band of both disks (Muto et al. 2012; Follette et al. 2013). This emission, likely optically thick, does not directly traces mass surface density. In SAO 206462 two spiral-like structures are seen in $\mathrm{H}$-band imaging (Figure 2). Muto et al. (2012) compared the shape of these structures with theoretical models for a perturbation due to low-mass companions, their best-fit spirals, S1 and S2, are labeled in Figure 2. For the S1 spiral, the location of the unseen perturber coincides with the location of the peak of emission in the ALMA map (at $\sim 55 \mathrm{AU}$ from the star with P.A. of $204^{\circ}$ ). If the $0.45 \mathrm{~mm}$ observations trace a mass overdensity at this location, we may be witnessing a spiral wave pattern that arose from a density enhancement rather than a point-like perturber, as reviewed by Paardekooper et al. (2010). Figure 2 also shows the location of S1/S2 with respect to the modeling residuals. Some overlap exists between the submillimeter residuals and the 
near-IR emission, with the S1/S2 spiral arms generally found inward of where the submillimeter residuals are. Nevertheless, observations with better angular resolution of SAO 206462 are required to investigate whether the submillimeter emission traces the spiral structures observed in the near-IR.

In the case of SR 21, Follette et al. (2013) showed that not only is the $H$-band polarized intensity image is rather smooth and axisymmetric, but it is also not compatible with the large depletion of material in the inner disk $\left(10^{-6}\right)$ found by Andrews et al. (2011). Furthermore, radiative transfer modeling of these near-IR observations did not find a transition between the outer disk and the depleted inner disk, even for modest depletion levels of 1\%-10\% (Follette et al. 2013). These $H$-band observations of SR 21 show evidence for emission from small grains out to a 80 AU radius, and compared with our ALMA observations, it shows no signs of the observed asymmetries (Figure 3 ). The difference in spatial distribution of the small grains (traced by near-IR observations) and the mm-sized particles (traced by these ALMA observations), could arise from the presence of a local enhancement in the gas density. For example disk vortices, which can be excited by Rossby-wave instabilities at the outer edge of gas depleted cavities, are particularly efficient at trapping large dust grains while small grains remain coupled to the gas (Birnstiel et al. 2013). Although not as dramatic as in the Oph IRS 48 case, the vortex in SR 21 may be driving the distribution of large and small particles within the disk.

\section{CONCLUSIONS}

We have observed two transitional disks, SAO 206462 and SR 21, with high sensitivity using ALMA. These transitional disks display large-scale asymmetries in their dust continuum emission, just as two other recently observed with ALMA (Casassus et al. 2013; Fukagawa et al. 2013; van der Marel et al. 2013), and an additional disk observed with CARMA (Isella et al. 2013). It is interesting to note that of the few such objects observed with high sensitivity and high angular resolution, most exhibit high-contrast asymmetrical structures. The lack of previous detections is likely due to phase errors introduced by the atmosphere and the lack of sensitivity of previous observations. This may imply that the process (or processes) that drive these asymmetries is taking place in most transitional disks.

In the case of SAO 206462 and SR 21, we have demonstrated that a ring does not describe these observations, and a vortex-like asymmetry, in addition to a ring of emission, is a better match. Such asymmetrical structures encompass a significant fraction ( $\sim 20 \%$ ) of the total disk emission, although we found that their contrast is much smaller than in $\mathrm{LkH} \alpha 330$, HD 142527, and Oph IRS 48. Asymmetries in SAO 206462 and SR 21 have a narrow radial size and a broad azimuthal extent, similar to the LkH $\alpha 330$ and Oph IRS 48 asymmetrical structures. If these asymmetries are in fact non-transient vortices, the turbulence inside them needs to be substantial in order to produce the azimuthally broad asymmetrical features.

Despite being a better fit, the vortex prescription does not reproduce every observed feature. Significant residuals remain that are suggestive of spiral-like structure, in the case of SAO 206462 some residuals roughly coincide with the spiral arms seen in $H$-band scattered light. To test the presence of large-scale spiral-like features, and to make sure the observed structures trace the mass surface density of the disk down to the midplane, observations at longer wavelengths are required. Future observations with ALMA at $3 \mathrm{~mm}$, and/or with the Very Large Array at $7 \mathrm{~mm}$, will help in this regard, since such observations trace larger dust grains and will have lower optical depth.

We thank Takayuki Muto, Carol Grady, and Katherine Follette for providing the near-IR images, and we thank Wladimir Lyra for useful discussions. A.I., J.M.C., L.M.P acknowledge support from NSF award AST-1109334. The National Radio Astronomy Observatory is a facility of the National Science Foundation operated under cooperative agreement by Associated Universities, Inc. This Letter makes use of the following ALMA data: ADS/JAO.ALMA 2011.0.00724.S. ALMA is a partnership of ESO (representing its member states), NSF (USA) and NINS (Japan), together with NRC (Canada) and NSC and ASIAA (Taiwan), in cooperation with the Republic of Chile. The Joint ALMA Observatory is operated by ESO, AUI/NRAO and NAOJ.

Facility: ALMA

\section{REFERENCES}

Andrews, S. M., Wilner, D. J., Espaillat, C., et al. 2011, ApJ, 732, 42 Andrews, S. M., Wilner, D. J., Hughes, A. M., Qi, C., \& Dullemond, C. P. 2009, ApJ, 700, 1502

Birnstiel, T., Dullemond, C. P., \& Pinilla, P. 2013, A\&A, 550, L8

Beckwith, S. V. W., Sargent, A. I., Chini, R. S., \& Guesten, R. 1990, AJ, 99, 924

Brown, J. M., Blake, G. A., Dullemond, C. P., et al. 2007, ApJL, 664, L107

Brown, J. M., Blake, G. A., Qi, C., et al. 2009, ApJ, 704, 496

Brown, J. M., Pontoppidan, K. M., van Dishoeck, E. F., et al. 2013, ApJ, 770,94

Bruderer, S. 2013, A\&A, 559, A46

Casassus, S., van der Plas, G., M, S. P., et al. 2013, Natur, 493, 191

Clarke, C. J., Gendrin, A., \& Sotomayor, M. 2001, MNRAS, 328, 485

Dullemond, C. P., \& Dominik, C. 2005, A\&A, 434, 971

Dutrey, A., Guilloteau, S., Piétu, V., et al. 2008, A\&A, 490, L15

Espaillat, C., D’Alessio, P., Hernández, J., et al. 2010, ApJ, 717, 441

Follette, K. B., Tamura, M., Hashimoto, J., et al. 2013, ApJ, 767, 10

Foreman-Mackey, D., Hogg, D. W., Lang, D., \& Goodman, J. 2013, PASP, 125,306

Fukagawa, M., Tsukagoshi, T., Momose, M., et al. 2013, PASJ, 65, L14

Garufi, A., Quanz, S. P., Avenhaus, H., et al. 2013, A\&A, 560, 105

Hernández, J., Hartmann, L., Megeath, T., et al. 2007, ApJ, 662, 1067

Ireland, M. J., \& Kraus, A. L. 2008, ApJL, 678, L59

Isella, A., Pérez, L. M., \& Carpenter, J. M. 2012, ApJ, 747, 136

Isella, A., Pérez, L. M., Carpenter, J. M., et al. 2013, ApJ, 775, 30

Lombardi, M., Lada, C. J., \& Alves, J. 2008, A\&A, 480, 785

Lyo, A.-R., Ohashi, N., Qi, C., Wilner, D. J., \& Su, Y.-N. 2011, AJ, 142, 151

Lyra, W., \& Lin, M.-K. 2013, ApJ, 775, 17

Müller, A., van den Ancker, M. E., Launhardt, R., et al. 2011, A\&A, 530, A85

Muto, T., Grady, C. A., Hashimoto, J., et al. 2012, ApJL, 748, L22

Paardekooper, S.-J., Lesur, G., \& Papaloizou, J. C. B. 2010, ApJ, 725, 146

Pontoppidan, K. M., Blake, G. A., van Dishoeck, E. F., et al. 2008, ApJ, 684,1323

Regály, Z., Juhász, A., Sándor, Z., \& Dullemond, C. P. 2012, MNRAS, 419, 1701

Roeser, S., Demleitner, M., \& Schilbach, E. 2010, AJ, 139, 2440

Salyk, C., Blake, G. A., Boogert, A. C. A., \& Brown, J. M. 2009, ApJ, 699,330

Simon, J. B., Armitage, P. J., \& Beckwith, K. 2011, ApJ, 743, 17

van der Marel, N., van Dishoeck, E. F., Bruderer, S., et al. 2013, Sci, 340, 1199

Wolf, S., \& D’Angelo, G. 2005, ApJ, 619, 1114 